\title{
Inflammation triggers specific microRNA profiles in human adipocytes and macrophages and in their supernatants
}

\author{
Francisco José Ortega ${ }^{1,2^{*}}$, María Moreno ${ }^{1}$, Josep María Mercader ${ }^{3}$, José María Moreno-Navarrete ${ }^{1,2}$, \\ Núria Fuentes-Batllevell ${ }^{1}$, Mònica Sabater ${ }^{1}$, Wifredo Ricart ${ }^{1,2}$ and José Manuel Fernández-Real ${ }^{1,2^{*}}$
}

\begin{abstract}
Background: The relevance of microRNAs (miRNAs) in adipose tissue is increasingly recognized, being intrinsically linked to different pathways, including obesity-related inflammation. In this study, we aimed to characterize the changes induced by inflammation on the miRNA pattern of human adipocytes and macrophages. Therefore, an extensive profile of 754 common miRNAs was assessed in cells (human primary mature adipocytes, and the macrophage-like cell line THP-1) and in their supernatants (SN) using TaqMan low-density arrays. These profiles were evaluated at the baseline and after administration of lipopolysaccharide (LPS, $10 \mathrm{ng} / \mathrm{ml}$ ) and LPS-conditioned medium from M1 macrophages (MCM, 5\%). The miRNAs that experienced the most dramatic changes were studied in subcutaneous human adipose tissue before and approximately 2 years after bariatric surgery-induced weight loss.

Results: Differentiated adipocytes expressed 169 miRNAs, being 85 detectable in the SN. In M1 macrophages, 183 miRNAs were detected, being 106 also present in the SN. Inflammation led to an increased number of miRNAs detectable in cells and in their SNs in both adipocytes $(+8.3 \%$ and $+24.7 \%)$ and $\mathrm{M} 1$ macrophages $(+1.4 \%$ and $+5 \%$, respectively). Indeed, under inflammatory conditions, adipocytes and M1 macrophages shared the expression of $147(+9 \%)$ miRNAs, and 100 (+41\%) common miRNAs were found in their SNs. Twelve of these factors were also linked to inflammation in whole adipose tissue from obese subjects. Interestingly, miR-221 (2-fold, $P=0.002$ ), miR-222 (2.5-fold, $P=0.04)$, and miR-155 (5-fold, $P=0.015)$ were increased in inflamed adipocytes and in their SNs (15-, 6-, and 4-fold, respectively, all $P<0.001)$. Furthermore, their expressions in human adipose tissue concordantly decreased after weight loss $(-51 \%, P=0.003,-49 \%, P=0.03$, and $-54.4 \%, P=0.005$, respectively).
\end{abstract}

Conclusions: Inflammation induces a specific miRNA pattern in adipocytes and M1 macrophages, with impact on the physiopathology of obesity-induced inflammation of adipose tissue. The crosstalk between cells should be investigated further.

Keywords: microRNAs, RT-PCR, Profiling, Macrophages, Adipocytes, Adipose tissue, Obesity, Inflammation

\section{Background}

Obese adipose tissue (AT) is a critical player in chronic low-grade systemic inflammation, with impact on insulin resistance through the secretion of hormones, adipocytokines [1], fatty acids [2], and (potentially) microRNAs [3] that modulate different metabolic pathways in AT itself, liver, skeletal muscle, and in the vasculature [4].

\footnotetext{
* Correspondence: fortega@idibgi.org; jmfreal@idibgi.org

'Department of Diabetes, Endocrinology and Nutrition (UDEN), Institut d'Investigació Biomédica de Girona (Id|BGi), Avinguda de França s/n, 17007 Girona, Spain

Full list of author information is available at the end of the article
}

Differentiated adipocytes release a great amount of inflammatory cytokines and chemokines (the so-called adipokines) in response to activated macrophages $[5,6]$. Weight loss is known to result in reduced macrophage infiltration in parallel to decreased expression of proinflammatory genes $[7,8]$. Many of these factors are able to maintain sustained subclinical inflammation in obese AT, and activate other immune cells $[9,10]$.

MicroRNAs (miRNAs) are non-coding RNAs that modulate gene expression through translational repression of specific target genes [11]. Many of these small

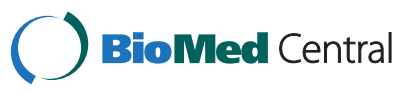

(c) 2015 Ortega et al.; licensee Biomed Central. This is an Open Access article distributed under the terms of the Creative Commons Attribution License (http://creativecommons.org/licenses/by/4.0), which permits unrestricted use, distribution, and reproduction in any medium, provided the original work is properly credited. The Creative Commons Public Domain Dedication waiver (http://creativecommons.org/publicdomain/zero/1.0/) applies to the data made available in this article, unless otherwise stated. 
RNAs are found in human AT, being differentially expressed in obese AT [12]. The close association between the miRNA expression profile in tissues and metabolic diseases such as obesity and type 2 diabetes is being increasingly recognized [13]. Several miRNAs are involved in adipocyte differentiation [14] and fat cell behavior [15]. So far, conditional disruption of Dicer, a key miRNA processing, may lead to impaired adipogenesis and fat mass shrinkage [16], reinforcing the relevance of miRNAs in AT development, inflammation, and metabolism. Not only tissue miRNAs play an important role, since specific circulating miRNA profiles exist in plasma from obese subjects [17], suggesting that some of these factors may modulate the systemic metabolic response. In this study, we aimed to evaluate the main effects of inflammation on the miRNA profile of differentiated human adipocytes, the macrophage-like cell line THP-1 (M1), and in their supernatants (SNs). Then, the miRNAs that experienced the most dramatic changes were evaluated in human subcutaneous AT before and after bariatric surgery-induced weight loss and the subsequent metabolic improvement and decreased inflammation.

\section{Results}

Comprehensive miRNA profiling in macrophages and adipocytes

We measured the expression (cells) and the presence into the supernatant (SN) of 754 common mature miRNAs in differentiated human subcutaneous adipocytes and the macrophage-like THP-1 cell line (M1) at the baseline and upon inflammation (Figure 1). Differentiated adipocytes expressed 169 miRNAs $(22.3 \%$ of the tested mature miRNAs), and 85 (11.2\%) of these factors were found in the SN (Figure 1 and Additional file 1). Interestingly, changes regarding miRNA expression patterns and their concentration in SN clearly identified the inflammatory effects in isolated mature adipocytes cultured and differentiated in vitro (Figure 1). Indeed, some miRNAs became undetectable (miR-1274B, miR572, and miR-766), while others appeared de novo in cells (miR-140-5p, miR-222\%, miR-376c, miR-411, and miR-146a) and their SNs (miR-146a, miR-146b, miR19a, miR-223*, miR-425, and miR-9*) upon MCMinduced inflammation (Figure 2). Overall, treatment with MCM increased the number of miRNAs expressed

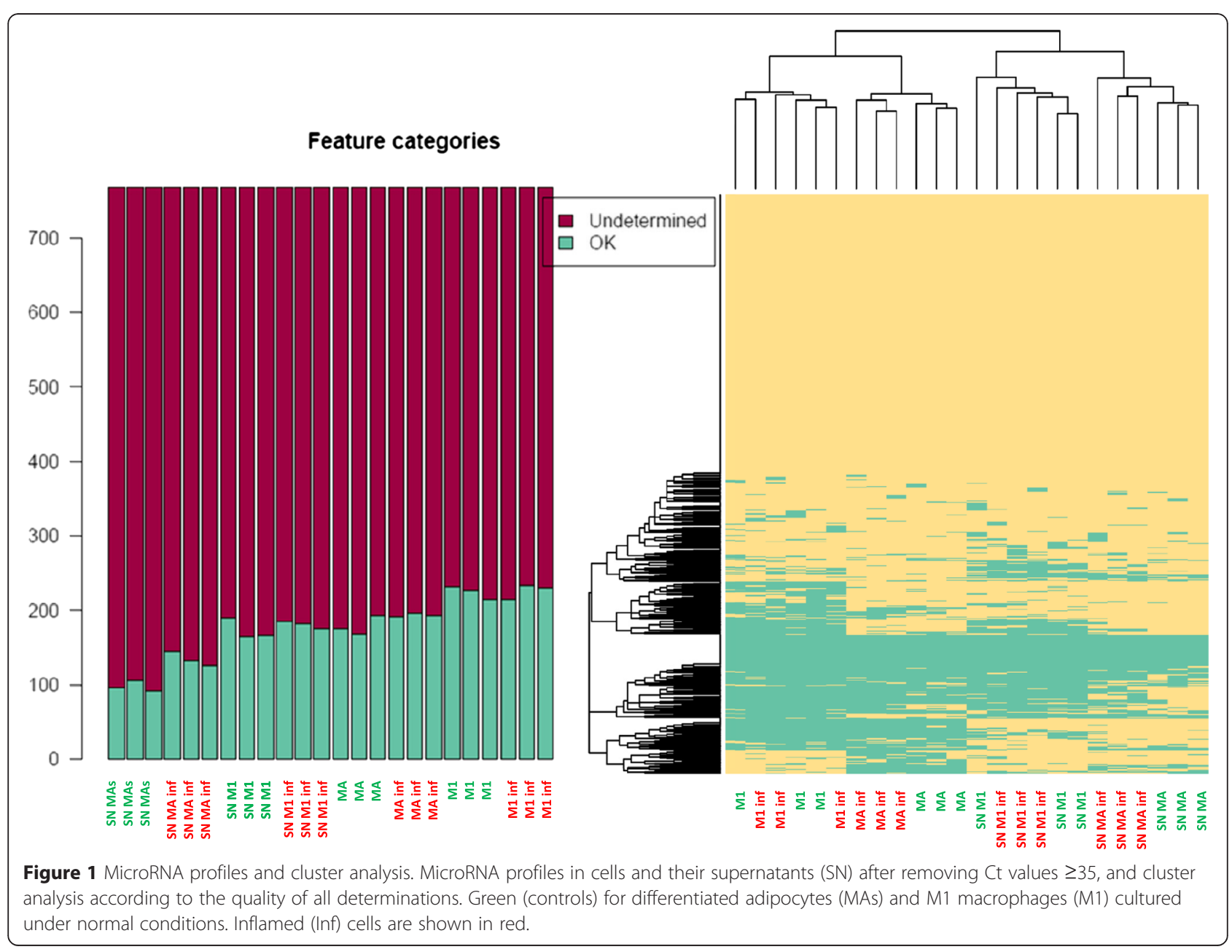




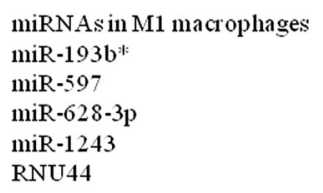

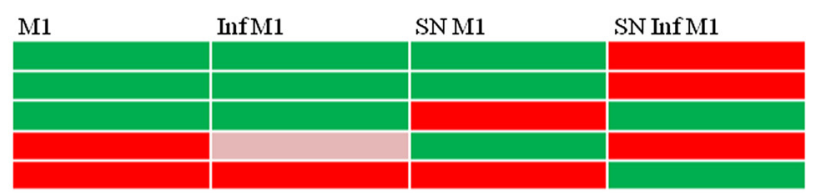

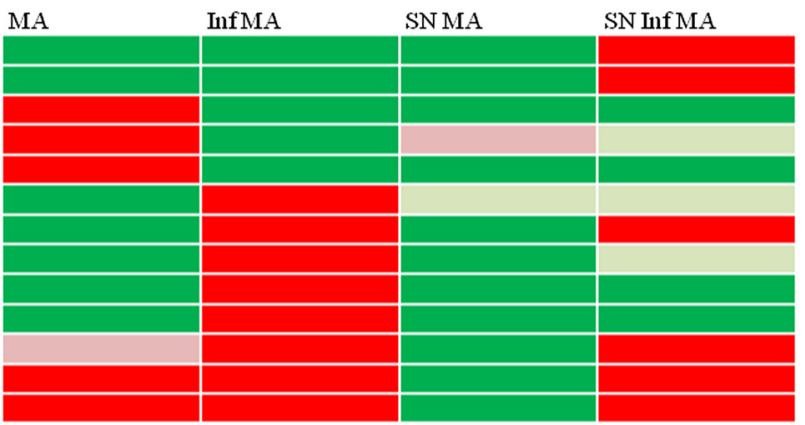

\begin{tabular}{ll|l|l|l|l|} 
noreplicates with $<35$ Cts & $\mathbf{0}$ & $\mathbf{1}$ & $\mathbf{2}$ & $\mathbf{3}$ \\
\hline
\end{tabular}

Figure 2 Major variations in miRNA profiles. Major variations in miRNA detection in cells and supernatants (SN) upon inflammation (Inf).

in adipocytes $(+8.3 \%)$ and in their SN $(+24.7 \%)$, concomitantly with increased $I L-6$ (79-fold change, $P=0.004)$ and TNF $\alpha$ (14-fold change, $P<0.0001)$ gene expression (Table 1).

On the other hand, expression of 183 miRNAs (24\%) and the presence in the SN of 106 (14\%) were detected in M1 macrophages. Inflammatory stimuli with LPS increased the number of miRNAs expressed $(+1.4 \%$ with respect to basal) and found in the SN (+5\%) of differentiated M1 macrophages. Among them, miR-628-3p and RNU44 (detectable in SN at the basal level but not after treatment with LPS) and miR-1243, miR-193b*, and miR-597 (detectable in SN upon inflammation, Figure 2) changed their detection levels after stimulation. However, these qualitative changes did not reach significance enough to discriminate activated cells from controls (Figure 1). Indeed, the inflammatory reaction of LPSstimulated M1 macrophages, as inferred from changes in the expression of well-recognized pro-inflammatory genes such as IL-6 and TNF $\alpha$ (17.7-fold change, $P=$ 0.002 , and 4.9 -fold change, $P=0.001$, respectively), did not show such great impact, when compared to the changes accounted in mature adipocytes upon inflammation (Table 1). The lack of major differences in stimulated M1 macrophages was more probably due to the transient basal inflammatory state that the induction of the mature type 1 macrophage-like state M1 (by treating THP-1 cells with phorbol 12-myristate 13-acetate) promotes per se. Nonetheless, under basal conditions adipocytes and M1 macrophages shared expression of 135, and 71 common mature miRNAs were found in SNs from both cell lines. Upon inflammatory stimuli, differentiated adipocytes and isolated M1 macrophages expressed 147 (+9\%) common mature miRNA species, and $100(+41 \%)$ of these factors were found in the SN (Additional file 2: Table S1).

\section{Quantitative changes upon inflammation}

When comparing normalized values of quantifiable miRNAs (Cts $<35$ in all replicates) either in cells or the SNs of M1 macrophages and isolated adipocytes following or not treatment (inflammation vs. control), significant changes in 18 mature miRNAs were identified in mature adipocytes and 24 in M1 macrophages (Table 1). Among them, increased expression of miR-221 (2-fold, $P=0.002)$, miR-222 (2.5-fold, $P=0.04)$, and miR-155 (5-fold, $P=0.015$, Figure 3 ) was found in inflamed adipocytes, as well as in the SN (15-, 6-, and 4-fold changes, respectively, all with $P$ values under 0.001 , Figure 3), when compared to control. Other significant changes in isolated adipocytes included increased expression of miR-132, miR-17, miR-31, miR-93, and miR-345 and decreased miR-422a expression. In the SN, increased concentrations of miR-19b, miR-92a, miR-342-3p, miR223, and miR-20a and decreased miR-199a-3p, miR-214, miR-193b, and miR-152 were also identified (Table 1).

On the other hand, increased expressions of miR-155 (2.5-fold, $P=0.001)$ but decreased miR-221 (0.49-fold, $P=0.034)$ were found in LPS-stimulated M1 macrophages (Table 1). Although no significant variations were identified in miR-222, modulation of miR-19a (0.5fold, $P=0.032$, and 2-fold, $P=0.034$ ) and decreased miR145 in both cells and SNs $(0.2$-fold, $P=0.005$, and 0.14 -fold, $P=0.032$ ) were shown in M1 macrophages reacting to LPS (Table 2). Other relevant changes in macrophages included increased miR-146a (5-fold change, $P<0.0001$ ), and the 
Table 1 Significant modulation of miRNAs expressed and/ or secreted (supernatant) by $\mathbf{M} 1$ macrophages upon lipopolysaccharide (LPS, $10 \mathrm{ng} / \mathrm{ml}$ ) stimuli and mature adipocytes treated or not with macrophages LPS-conditioned medium (MCM, 5\%)

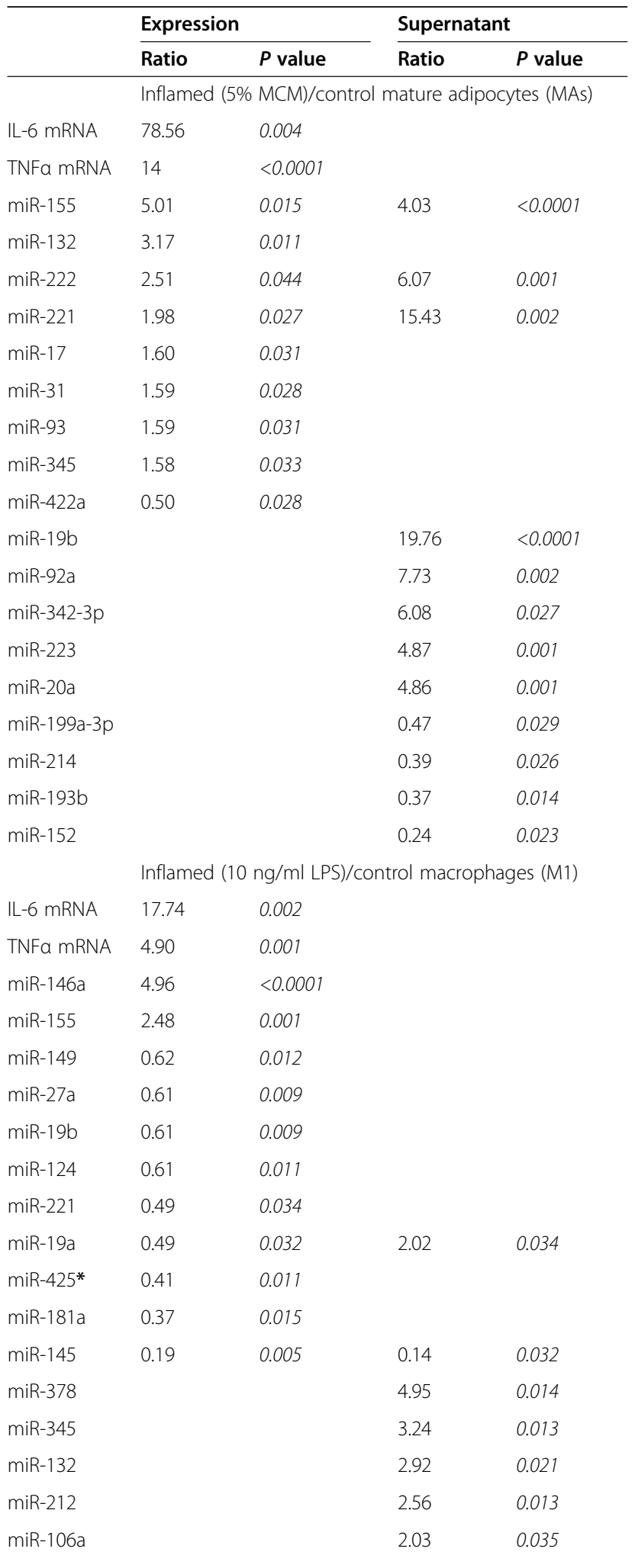

Table 1 Significant modulation of miRNAs expressed and/ or secreted (supernatant) by M1 macrophages upon lipopolysaccharide (LPS, $10 \mathrm{ng} / \mathrm{ml}$ ) stimuli and mature adipocytes treated or not with macrophages LPS-conditioned medium (MCM, 5\%) (Continued)

\begin{tabular}{lll}
\hline miR-25 & 1.93 & 0.016 \\
miR-24 & 1.77 & 0.005 \\
miR-92a & 1.75 & 0.007 \\
miR-30a-5p & 1.55 & 0.010 \\
miR-30e-3p & 0.39 & 0.002 \\
RNU48 & 0.22 & 0.015 \\
miR-15b & 0.20 & 0.037 \\
let-7a & 0.12 & 0.014
\end{tabular}

Results assessed were compared by Student $t$-test. Italics for significant results.

decreased expression of nine specific mature miRNAs, which were superseded by increased presence in the $\mathrm{SN}$ of another set of nine different miRNAs (Table 1).

\section{In vivo validation}

Noteworthy, qRT-PCR analyses in AT from morbid obese subjects following approximately 2 years of weight loss identified significant changes in 12 of 18 miRNAs (67\%) shortlisted by analyses in vitro (Table 2). Currently, it is well recognized that, when compared with conventional strategies, the physiological impact of bariatric surgery is of particular interest, with regard to short-term weight loss and improvement of inflammation [18]. Indeed, concomitantly with weight loss and improved AT inflammation (Additional file 2: Table S2), current data pointed decreased AT expression of miRNAs which detection inside isolated cells (for example, miR-146b, miR-376c, miR-411) and/or in the SN (miR221, miR-222, miR-155, miR-223, miR-19a/b) rose in differentiated adipocytes upon inflammatory stimuli (Table 2). On the other hand, some miRNAs highlighted by either differential expression or concentrations in the $\mathrm{SN}$ of inflamed M1 macrophages, such as miR-146a, miR-155, and miR-19a, also showed decreased expression in human obese AT after weight loss (Table 2).

\section{Discussion}

Current results indicate that inflammation alters miRNA profiles in adipocytes and macrophages. Adipose tissue (AT) exhibits inflammation as tissue mass expands in obesity, involving macrophage infiltration and the inflammatory response of adipocytes [8]. Analyses of AT before and after weight loss have revealed a reduction in macrophage number and decreased expression of pro-inflammatory genes [7]. There is preliminary information about the potential involvement of post-transcriptional regulatory elements such as microRNAs (miRNAs) in this context [19]. Indeed, secreted miRNAs may target 


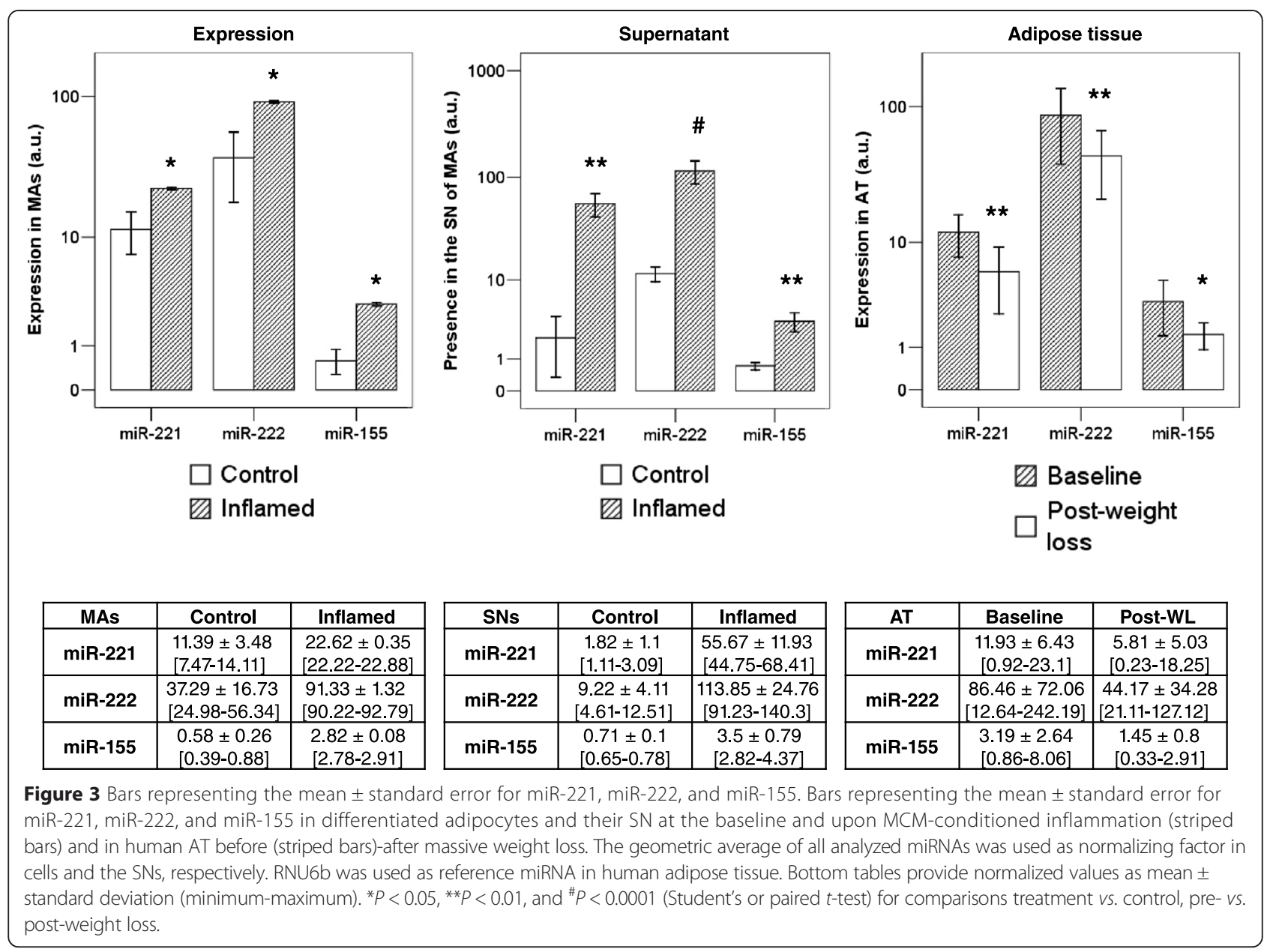

neighboring cells and perform regulatory functions in them $[3,19,20]$.

The most recent studies have revealed that plasma membrane-derived vesicles, namely exosomes and microvesicles, can transfer miRNAs from donor to recipient cells [21]. These extracellular vesicles are not simply 'garbage bags' or a route for exclusion of surplus molecules but active effectors in mediating adaptive responses in targeting cells [22]. Others have demonstrated that more extracellular vesicles containing miRNAs are released in ex vivo adipose tissue cultures of mice feeding a high-fat diet and of leptin-deficient obese mice when compared to control mice [23]. These exosomes released to the supernatant are predicted to have both local and systemic effects, being taken up by resident macrophages and leading to their activation in adipose tissue. Because of this activation, more macrophages may be recruited into AT to further increase the inflammatory response, with more miRNAs released from inflamed AT [23]. Also, HDL transports large amounts of endogenous miRNAs, being suggested as the possible mechanism whereby some of the biological effects of HDL could be explained [3].
The molecular mechanisms of RNA-based cell-to-cell communication and the exact process of how extracellular vesicles and HDL are loaded with miRNAs have not been characterized yet and, thus, the pathways involved remain largely unknown. However, since significant differences regarding miRNAs contained in AT [14] and the circulation [17] of obese patients exist, expression and secretion by inflamed adipocytes and activated M1 macrophages might be attractive candidates in this field.

\section{Inflammation alters miRNA profiles in adipocytes and macrophages}

Upon inflammation, an increased number of miRNAs were detectable in stimulated adipocytes and M1 macrophages, when compared to the basal conditions. This increase was more noticeable when studying the miRNAs that are found in the supernatant (SN) of adipocytes. Identification of miR-146a and miR-146b in adipocytes upon inflammation (but not under basal conditions), for example, was consistent when analyzing both cells and SNs, decreasing in human AT after weight loss. This 
Table 2 Testing of inflammation-induced miRNA candidates identified in vitro in human subcutaneous adipose tissue (AT) samples

\begin{tabular}{|c|c|c|c|c|c|c|c|c|c|c|}
\hline & \multicolumn{4}{|c|}{ Inflamed (5\% MCM)/control adipocytes } & \multicolumn{4}{|c|}{ Inflamed (10 ng/ml LPS)/control macrophages } & \multirow{2}{*}{\multicolumn{2}{|c|}{$\begin{array}{l}\text { Obese AT/post-weight loss } \\
\text { Human AT }(n=9)\end{array}$}} \\
\hline & \multicolumn{2}{|c|}{ Expression } & \multicolumn{2}{|c|}{ Supernatant } & \multicolumn{2}{|c|}{ Expression } & \multicolumn{2}{|c|}{ Supernatant } & & \\
\hline & Ratio & $P$ value & Ratio & $P$ value & Ratio & $P$ value & Ratio & $P$ value & $\%$ change & $P$ value \\
\hline miR-146b & \multirow{2}{*}{\multicolumn{4}{|c|}{ Only detectable in the SN of inflamed MAs }} & & & & & -88 & $<0.0001$ \\
\hline miR-146a & & & & & 4.96 & $<0.0001$ & & & -69.6 & 0.002 \\
\hline miR-376c & \multicolumn{6}{|c|}{ Only detectable in inflamed MAs } & & & -78 & 0.002 \\
\hline miR-221 & \multirow[t]{2}{*}{1.98} & \multirow[t]{2}{*}{0.027} & 15.43 & 0.002 & \multirow[t]{2}{*}{0.49} & \multirow[t]{2}{*}{0.034} & & & -51.3 & 0.003 \\
\hline miR-223 & & & 4.87 & 0.001 & & & & & -74.3 & 0.004 \\
\hline miR-411 & \multicolumn{4}{|c|}{ Only detectable in inflamed MAs } & & & & & -62.2 & 0.004 \\
\hline miR-155 & 5.01 & 0.015 & 4.03 & $<0.0001$ & 2.48 & 0.001 & & & -54.4 & 0.005 \\
\hline miR-19b & & & 19.76 & $<0.0001$ & 0.61 & 0.009 & & & -72.7 & 0.006 \\
\hline miR-19a & \multicolumn{4}{|c|}{ Only detectable in the SN of inflamed MAs } & 0.49 & 0.032 & 2.02 & 0.034 & -73.4 & 0.008 \\
\hline RNU48 & & & & & & & 0.22 & 0.015 & -45 & 0.009 \\
\hline miR-222 & 2.51 & 0.044 & 6.07 & 0.001 & & & & & -48.9 & 0.028 \\
\hline miR-193b & & & 0.37 & 0.014 & & & & & -44.8 & 0.039 \\
\hline miR-766 & \multicolumn{4}{|c|}{ Undetectable in inflamed MAs } & & & & & 182 & 0.084 \\
\hline let-7a & & & & & & & 0.12 & 0.014 & 6.1 & 0.276 \\
\hline miR-145 & & & & & 0.19 & 0.005 & 0.14 & 0.032 & -26.9 & 0.228 \\
\hline miR-92a & & & 7.73 & 0.002 & & & 1.75 & 0.007 & -21.3 & 0.327 \\
\hline miR-572 & \multirow{2}{*}{\multicolumn{4}{|c|}{ Undetectable in inflamed MAs }} & & & & & -7.6 & 0.413 \\
\hline miR-378 & & & & & & & 4.95 & 0.014 & -41 & 0.78 \\
\hline
\end{tabular}

Results from nine morbid obese participants before and after surgery-induced weight loss (post-weight loss, see Additional file 2: Table S2 for additional details). Results assessed were compared by paired $t$-test. 'Detectable' refers to $<35 \mathrm{Cts}$, while 'undetectable' indicates $\geq 35 \mathrm{Cts}$ in all biological replicates ( $n=3$ ), as explained above. Italics for significant results.

agrees with the notion that AT miR-146 increases as fat mass expands in mice models of obesity [24]. On the other hand, the expression of 'miRNokines' such as miR146b, miR-376c, miR-411, and miR-19a by inflamed adipocytes/obese AT may hint into functional activities beyond adiposity, such as recruitment of immune cells [25], or modulation of insulin-secreting cells [26]. For instance, miR-19b directly regulates the formation of 'foam cells' derived from macrophages [27] and was measurable here in the SN of LPS-activated M1 macrophages (but not in controls). This may suggest mechanisms by which cells release a particular set of miRNAs to the $\mathrm{SN}$ and miRNAs which are more likely to be kept inside cells. Further investigations are needed in order to determine these mechanisms.

In differentiated adipocytes, miR-221, miR-222, and miR-155 were detectable and measurable in both cells and SNs, being their values significantly increased upon inflammation. These three miRNA candidates were also associated with obesity and weight loss, when analyzed in AT samples. Indeed, this increased expression in obese AT has been reported previously [24,14], as well as their association with AT oxidative stress, apoptosis, and inflammatory events [28]. In agreement with current results, macrophages in atherosclerotic lesions exhibit increased expression of miR-155, which also responds to inflammatory cues [29]. On the other hand, TNF $\alpha$ induced the expression of both miR-221 and miR-222, which were also found to be downregulated during adipogenesis and upregulated in obese mice [30]. The concordant and sustained changes in the expression of these three miRNAs seem to be connected with inflammatory nodes in obese AT, suggesting their participation in regulatory networks underlying adipogenesis and AT dysfunction. In this respect, three major target genes [c-Cbl protein, phosphoinositide-3-kinase regulatory subunit 1 (PI3KR1), and the suppressor of cytokine signaling 1 (SOCS1)] for the in silico intersection between two of the more relevant miRNAs in the context of inflammation affecting fat cells, the miR-155 and miR221 (Additional file 1: Table S3), are involved in insulin pathway (Additional file 3: Figure S2) and, thus, may participate of insulin resistance and type 2 diabetes (Additional file 3: Figure S3) [31]. The PIK3R1 gene, encoding the $\mathrm{p} 85$ regulatory subunit of phosphatidylinositol 3-kinase (PI3K), couples insulin and leptin signaling pathways, playing a critical role in mediating AT insulin sensitivity, and the initiation and propagation of the 
inflammatory response [32]. Also, the proto-oncogene Casitas b-lineage lymphoma (CBL) plays an important role in AT hyperplasia and insulin action, independently of the PI3K/Akt pathway [33]. The ability of insulin to stimulate glucose uptake relies on a complex signaling cascade that leads to the translocation of glucose receptors to the membrane. Thus, the modulation of expression and secretion of these miRNAs in AT, particularly in adipocytes, may contribute to the improvement of insulin sensitivity following massive fat mass loss and decreased inflammation. Understanding the miRNA profiles associated with different pathways for macrophage activation could also provide a new spectrum of biomarkers for identification and a potential therapeutic approach to mitigate entry into tissues and the onset of inflammatory diseases.

miRNA profiles in macrophages and adipocytes and their SNs Type 1 macrophage-like human monocytes and human subcutaneous differentiated adipocytes share expression of several miRNAs, being this expression enhanced in the former. The data agrees with accumulating evidence that adipocytes and immune cells show common features, with expression of genes previously considered to be specific of one lineage [34], and the concept that proinflammatory components are not only secreted by the stromal-vascular cell fraction but also by differentiated adipocytes [35]. Notably, when comparing inflamed adipocytes and M1 macrophages, the number of miRNAs that were only detectable in adipocytes or in macrophages decreased, especially when analyzing their SNs. Thus, the similitude between mature adipocytes and macrophages [36] becomes clearer through concomitant changes in miRNA expression pattern and the miRNAs found in the SN when inflammation appears.

\section{Conclusions}

Current results highlight the existence of cell-dependent miRNA profiles inside and in the SN of isolated adipocytes and M1 macrophages. Obesity-related inflammation increases the production of miRNAs and alters the expression pattern in both cells and SNs. Understanding miRNA profiles associated with different pathways of adipocytes and macrophages activation may provide a new spectrum of biomarkers and therapeutic approaches to mitigate macrophage infiltration in obese AT and the onset of obesity-associated inflammation. Variations regarding miR-221/222 and miR-155, for example, may participate in the crosstalk between obesity-related inflammation, insulin resistance, and other obesity-associated morbidities. This work paves the way for future clinical and cellular studies aimed at determining the impact of these molecular adaptations on the development of obesityrelated inflammation and such deleterious effects.

\section{Methods}

\section{Cell culture}

The human monocyte cell line THP-1 (ATCC) was cultured in RPMI 1640 medium containing 10\% fetal bovine serum, $5 \mathrm{mM}$ glucose, $2 \mathrm{mM}$ L-glutamine, $50 \mathrm{mg} / \mathrm{ml}$ gentamicin, and $20 \mathrm{mM}$ HEPES at $37^{\circ} \mathrm{C}$ in a humidified $5 \%$ $\mathrm{CO}_{2}$ and $95^{\circ} \mathrm{C}$ air atmosphere. The mature type 1 macrophage-like state (M1) was induced by treating THP-1 cells with $0.162 \mathrm{mM}$ of phorbol 12-myristate 13-acetate (PMA) for $24 \mathrm{~h}$. Differentiated, plastic-adherent cells were washed with cold Dulbecco's phosphate-buffered saline (Sigma Chemical Co., St. Louis, MO, USA) and incubated with fresh medium without PMA. Differentiated M1 macrophages were treated with fresh medium and fresh medium containing $10 \mathrm{ng} / \mathrm{ml}$ of lipopolysaccharides (LPS, Sigma Chemical Co.) for an additional 24-h period (three biological replicates per treatment), as previously described [5]. Then, the medium was collected and centrifuged at $400 \cdot g$ for 5 min, diluted with adipocyte medium (Zen-Bio, Inc., Research Triangle Park, NC, USA), and used as macrophage LPS-conditioned media (MCM, 5\%) to induce inflammation in differentiated human adipocytes (MAs).

Human subcutaneous preadipocytes from a non-diabetic Caucasian male with body mass index (BMI) $<30$ and age $<40$ (Zen-Bio, Inc.) were cultured with the Preadipocytes Medium (PM, Zen-Bio, Inc.) in a humidified $37^{\circ} \mathrm{C}$ incubator with $5 \% \mathrm{CO}_{2}$ and $95^{\circ} \mathrm{C}$ air atmosphere. Twenty-four hours after plating, cells were checked for confluence and differentiated using the commercially available Differentiation Medium (DM, Zen-Bio, Inc.) following manufacturer's instructions. Two weeks after initializing differentiation, cells appeared rounded with large lipid droplets in the cytoplasm. Cells were considered mature adipocytes and incubated with fresh adipocytes media (control) or fresh media containing 5\% $\mathrm{MCM}$, to mimic the inflammatory milieu of obese AT. After $24 \mathrm{~h}$ of treatment, the supernatants were centrifuged at $400 \cdot g$ for $5 \mathrm{~min}$, and both pellets and supernatants were removed and stored at $-80^{\circ} \mathrm{C}$ for future analysis. Three biological replicates per treatment were performed and analyzed (Additional file 3: Figure S1 - Workflow diagram).

\section{Subject recruitment}

Expression and validation of the miRNAs shortlisted in vitro was assessed in subcutaneous abdominal adipose tissue (AT) from nine morbidly obese women before and after approximately 2 years of surgery-induced weight loss $\left(\mathrm{BMI}=43.4 \pm 5 \mathrm{~kg} / \mathrm{m}^{2}\right.$, age $=48 \pm 10$ years [mean \pm $\mathrm{SD}$ ], Additional file 2: Table S2). These volunteers were recruited at the Department of Diabetes, Endocrinology and Nutrition and the Department of Surgery of the Hospital 'Dr Josep Trueta' of Girona (Girona, Spain). All subjects provided written informed consent before entering the study, were of Caucasian origin, and reported 
that their body weight was stable for at least 3 months before entering the study. No systemic diseases other than obesity were reported. All participants were free of any infections in the previous month before entering the study. Liver and thyroid dysfunction were specifically excluded by biochemical work-up. Other exclusion criteria included the following: 1) clinically significant hepatic, neurological, or other major systemic disease, including malignancy, 2) history of drug or alcohol abuse or serum transaminase activity more than twice the upper limit of normal, 3) an elevated serum creatinine concentration, 4) acute major cardiovascular event in the previous 6 months, 5) acute illnesses and current evidence of highgrade chronic inflammatory or infective diseases, and 6) mental illness rendering the subjects unable to understand the nature, scope, and possible consequences of this study. The study protocol was approved by the Ethics Committee and the Committee for Clinical investigation (CEIC) of the Hospital 'Dr. Josep Trueta' of Girona, so we certify that all applicable institutional regulations concerning the ethical use of information and samples from human subjects were followed during this research.

\section{RNA extraction}

Total RNA, including small RNA species such as miRNAs, was extracted and purified from AT and cells (macrophages and adipocytes) using miRNeasy ${ }^{\circ}$ Mini Kit (QIAGEN, Gaithersburg, MD, USA). AT samples (approximately $150 \mu \mathrm{g}$ ) and cells were homogenized in $0.6 \mathrm{~mL}$ of $\mathrm{QIAzol}^{\circ}$ Lysis Reagent (QIAGEN), a monophasic solution of phenol and guanidine thiocyanate which facilitates sample disaggregation and inhibits RNAses. After addition of chloroform (0.4 volumes), the homogenate is separated into aqueous and organic phases by centrifugation $\left(15 \mathrm{~min}\right.$ at $12,000 \cdot g$ and $4^{\circ} \mathrm{C}$ ). Then, the upper aqueous RNA-rich phase was isolated and ethanol absolute (1.5 volumes) was added to provide appropriate binding conditions for all RNA molecules, including miRNAs. The sample was applied to a silica-membrane RNeasy spin columns, where RNA binds to the membrane while phenols and other compounds are washed away. High-quality RNA was finally eluted in $30 \mu \mathrm{L}$ of RNAse-free water. Final RNA concentrations were assessed with a Nanodrop ND-1000 Spectrophotometer (Thermo Fischer Scientific, Wilmington, DE, USA). The integrity was checked with the Nano lab-ona-chip assay for total eukaryotic RNA using Bioanalyzer 2100 (Agilent Technologies, Palo Alto, CA, USA).

miRNA profiles were assessed in cell supernatant (SN) following standard procedures previously optimized for plasma [17]. First, floating cells were removed from the media by an additional centrifugation of $2,000 \cdot g$ for 5 min at $4^{\circ} \mathrm{C}$ using a laboratory centrifuge (Beckman J-6M Induction Drive Centrifuge, Palo Alto, CA, USA). RNA extraction from cell media was performed using the mirVana PARIS Isolation Kit (Applied Biosystems, Darmstadt, Germany), as previously described [17].

\section{Retrotranscription to CDNA and pre-amplification}

A 2,000 ng of total RNA from AT and cell debris and a fixed volume of $3 \mu \mathrm{L}$ of RNA solution from the $40-\mu \mathrm{L}$ eluate of RNA isolation performed in SN were used as input into the reverse transcription (RT), using the TaqMan miRNA Reverse Transcription Kit and TaqMan miRNA Multiplex RT Assays, which are required to run the TaqMan Array microRNA Cards (Applied Biosystems). Pre-amplification was performed in RNA samples from SN using TaqMan PreAmp Master Mix and Megaplex ${ }^{\mathrm{Tt}}$ PreAmp Primers (human pool set A and B), which provided an optional amplification step prior to real-time analysis when the RNA sample is limiting. This step is mandatory to provide reliable results in the $\mathrm{SN}$, as well as in plasma [17].

\section{miRNA profiling using TLDAs}

TaqMan low-density arrays (TLDAs) for miRNAs, covering a total amount of 754 miRNA species, were applied to three biological replicates. Real-time (RT)-PCR was carried out on an Applied Biosystems 7900HT thermocycler. Data were analyzed with SDS Relative Quantification Software version 2.2.2 (Applied Biosystems), with an assigned minimum threshold above the baseline of all assays showing measurable amplifications above background and threshold thermal cycles (Cts) below 35 . The geometric average of all analyzed miRNAs was used as normalizing factor in cells and the SN. Normalized relative $\log _{10}$ ratios were used for posterior statistical tests, as previously described [37].

\section{Analysis of individual miRNAs using TaqMan hydrolysis probes}

Commercially available TaqMan hydrolysis probes (Applied Biosystems) were used to assess the expression of individual miRNAs in human AT. After RNA extraction and retrotranscription of 2,000 ng of RNA, the product was diluted $(1: 100)$ previous being combined $(5 \mu \mathrm{L})$ with $0.25 \mu \mathrm{L}$ of TaqMan miRNA hydrolysis probes $(20 \times)$ and $4.75 \mu \mathrm{L}$ of the LightCycler 480 Probes master mix $(2 \times)$ (Roche Diagnostics, Barcelona, Spain) to a final volume of $10 \mu \mathrm{L}$. Gene expression was assessed by real-time PCR using the LightCycler 480 Real-Time PCR System (Roche Diagnostics, Barcelona, Spain) and TaqMan technology suitable for relative gene expression quantification following manufacturer's protocol. RNU6b (NCBI Accession ID\#: NR_002752, assay ID\#: 4395470) was used as reference miRNA (or endogenous control) in human AT. 


\section{Gene expression}

Three micrograms of total RNA were reverse transcribed to cDNA using High-Capacity cDNA ${ }^{\oplus}$ Archive Kit (Applied Biosystems, Darmstadt, Germany) according to manufacturers' protocols. Commercially available and prevalidated $\mathrm{TaqMan}^{\circledR}$ primer/probe sets were used for gene expression determinations (Applied Biosystems). Expression was assessed by real-time PCR using the LightCycler $^{\circledR} 480$ Real-Time PCR System (Roche Diagnostics, Barcelona, Spain) and TaqMan ${ }^{\circledR}$ technology suitable for relative gene expression quantification. The reaction was performed following manufacturers' instructions in a final volume of $7 \mu \mathrm{l}$. The cycle program consisted of an initial denaturing of $10 \mathrm{~min}$ at $95^{\circ} \mathrm{C}$ then 45 cycles of 15 -s denaturizing phase at $92^{\circ} \mathrm{C}$ and $1-$ min annealing and extension phase at $60^{\circ} \mathrm{C}$. Then, the crossing point $(\mathrm{Cp})$ values were assessed for each amplification curve by the second derivative maximum method. $\Delta \mathrm{Cp}$ value was first calculated by subtracting the $\mathrm{Cp}$ value for corresponding endogenous controls in each sample from the $\mathrm{Cp}$ value for each sample and target gene. Fold changes compared with the endogenous control were then determined by calculating $2^{-\Delta C \mathrm{p}}$, so gene expression results are expressed as expression ratio relative to preselected and validated endogenous control. The commercially available and pre-validated TaqMan $^{\circledR}$ primer/probe sets used here for gene expression were as follows: interleukin 6 (IL-6, Hs00985639_m1), tumor necrosis factor (TNF $\alpha$, Hs01113624_g1), and leptin (LEP, Hs00174877_m1). Peptidyl-prolyl cis-trans isomerase A (PPIA, Hs99999904_m1, also known as cyclophilin A) expressions were assessed as endogenous control in each reaction and sample. Replicates and positive and negative controls were included.

\section{Statistical methods}

Tables were constructed for qualitative evaluation of the number and identity of miRNAs shared among all samples and cells. Tables for each group of treatments indicate the number of observed raw RT-PCR values below $35 \mathrm{Cts}$ in three biological replicates. The detectability of miRNA in cells or SN under different conditions was established on less than $35 \mathrm{Cts}$ for all replicates $(n=3)$. Regarding quantitative differences, normal distribution and homogeneity of variances were evaluated using Levene's test before conducting statistical analysis. Variables were given in a base $\log _{10}$ transformation and analyzed on that $\log _{10}$ scale when necessary. Student's or paired $t$-tests were performed to study differences between groups of samples. Data analyses were performed with the SPSS statistical software (SPSS v13.0, IBM, Chicago, IL, USA) and the $R$ Statistical Software (http:// www.r-project.org/). Potential miRNA target genes and pathways were identified using DIANA-mirPath [31] and TargetScan 6.0 [38].

\section{Additional files}

\begin{abstract}
Additional file 1: Supplemental information - raw data.
Additional file 2: Table S1. Most significant differences regarding miRNA availability in macrophages (M1) and adipocytes (MAs) and into supernatants (SNs) at the basal level and upon inflammation (Inf).

Table S2. Anthropometric and biochemical data and expression results in subcutaneous adipose tissue samples from nine participants recruited for validation in vivo before (baseline) and after (post-weight loss) bariatric surgery. Table S3. Intersection of in silico identified target genes of miR-155 and miR-221. 'Their involvement in insulin signaling is represented in Additional file 3: Figure S2 and S3.
\end{abstract}

Additional file 3: Figure S1. Workflow diagram. Figure S2. Insulin signaling pathway showing the involvement of CBL, SOCS1, and PIK3R1 (yellow boxes). Their post-transcriptional regulation may be accomplished by both miR-221 and miR-155. Figure S3. SOCS1 and PIK3R1 (yellow boxes) in the context of type II diabetes and impaired glucose intake.

\section{Competing interests}

The authors declare that they have no competing interests.

\section{Authors' contributions}

All authors of this manuscript have directly participated in the execution of the study. FJO wrote the manuscript, designed the study, participated in the analysis of biochemical variables, and performed the statistical analysis. MM, JMM, JMM-N, NF-B, and MS helped with the biochemical analyses. WR provided important intellectual content. JMF-R carried out the conception and coordination of the study, and contributed to writing the manuscript. All authors read and approved the final manuscript.

\section{Acknowledgements}

This study was supported by an EFSD/Lilly Fellowship award (FJ Ortega, 2013), by the Spanish Ministry of Science and Innovation (FIS 2011-00214, JM FernándezReal, 2011), Fondo Europeo de Desarrollo Regional (FEDER), and CIBER de la Fisiopatología de la Obesidad y la Nutrición (CIBERobn). The CIBERobn is an initiative from the Instituto de Salud Carlos III (ISCIII). Dr. Josep M Mercader was supported by a Sara Borrell fellowship, also from the Instituto de Salud Carlos III (ISCIII), and by an EFSD/Lilly Fellowship award. Dr. José M Fernández-Real is the guarantor for this work as a whole, including study design, access to data, and the decision to submit and publish the manuscript.

\section{The authors have nothing to disclose}

All authors of this manuscript have directly participated in the execution and analysis of the study. All authors are aware of and agree to the content of the manuscript, and all authors have approved the final version submitted and their being listed as an author on the manuscript. The contents of this manuscript have not been copyrighted or published previously. There are no directly related manuscripts or abstracts, published or unpublished, by one or more authors of this manuscript. The contents of this manuscript are not now under consideration for publication elsewhere. The submitted manuscript nor any similar manuscript, in whole or in part, will be neither copyrighted, submitted, or published elsewhere while the journal is under consideration.

\section{Author details}

${ }^{1}$ Department of Diabetes, Endocrinology and Nutrition (UDEN), Institut d'Investigació Biomédica de Girona (IdIBGi), Avinguda de França s/n, 17007 Girona, Spain. ${ }^{2}$ CIBER de la Fisiopatología de la Obesidad y la Nutrición (CIBERobn, CB06/03) and Instituto de Salud Carlos III (ISCIII), Sinesio Delgado 4, 28029 Madrid, Spain. 3 Joint BSC-CRG-IRB program on Computational Biology, Barcelona Supercomputing Center, Baldiri Reixac 10, 08028 Barcelona, Spain.

Received: 27 January 2015 Accepted: 7 April 2015

Published online: 24 April 2015

\section{References}

1. Tilg H, Moschen AR. Adipocytokines: mediators linking adipose tissue, inflammation and immunity. Nat Rev Immunol. 2006;6(10):772-83.

2. Delarue J, Magnan C. Free fatty acids and insulin resistance. Curr Opin Clin Nutr Metab Care. 2007;10(2):142-8. 
3. Vickers KC, Palmisano BT, Shoucri BM, Shamburek RD, Remaley AT. MicroRNAs are transported in plasma and delivered to recipient cells by high-density lipoproteins. Nat Cell Biol. 2011;13(4):423-33.

4. Clement K, Viguerie N, Poitou C, Carette C, Pelloux V, Curat CA, et al. Weight loss regulates inflammation-related genes in white adipose tissue of obese subjects. Faseb J. 2004;18(14):1657-69.

5. Bassols J, Ortega FJ, Moreno-Navarrete JM, Peral B, Ricart W, Fernandez-Real JM. Study of the proinflammatory role of human differentiated omental adipocytes. J Cell Biochem. 2009;107(6):1107-17.

6. Permana PA, Menge C, Reaven PD. Macrophage-secreted factors induce adipocyte inflammation and insulin resistance. Biochem Biophys Res Commun. 2006;341(2):507-14.

7. Cancello R, Henegar C, Viguerie N, Taleb S, Poitou C, Rouault C, et al. Reduction of macrophage infiltration and chemoattractant gene expression changes in white adipose tissue of morbidly obese subjects after surgery-induced weight loss. Diabetes. 2005;54(8):2277-86.

8. Weisberg SP, McCann D, Desai M, Rosenbaum M, Leibel RL, Ferrante Jr AW Obesity is associated with macrophage accumulation in adipose tissue. J Clin Invest. 2003;112(12):1796-808.

9. Meijer K, de Vries M, Al-Lahham S, Bruinenberg M, Weening D, Dijkstra M, et al. Human primary adipocytes exhibit immune cell function: adipocytes prime inflammation independent of macrophages. PLoS One. 2011;6(3):e17154.

10. Skurk T, Herder C, Kraft I, Muller-Scholze S, Hauner H, Kolb H. Production and release of macrophage migration inhibitory factor from human adipocytes. Endocrinology. 2005;146(3):1006-11.

11. Bartel DP. MicroRNAs: target recognition and regulatory functions. Cell. 2009;136(2):215-33.

12. Hilton C, Neville MJ, Karpe F. MicroRNAs in adipose tissue: their role in adipogenesis and obesity. Int J Obes (Lond). 2013;37(3):325-32.

13. Rottiers $V$, Naar AM. MicroRNAs in metabolism and metabolic disorders. Nat Rev Mol Cell Biol. 2012;13(4):239-50.

14. Ortega FJ, Moreno-Navarrete JM, Pardo G, Sabater M, Hummel M, Ferrer A, et al. MiRNA expression profile of human subcutaneous adipose and during adipocyte differentiation. PLoS One. 2010;5(2):e9022.

15. Oger F, Gheeraert C, Mogilenko D, Benomar Y, Molendi-Coste O, Bouchaer $E$, et al. Cell-specific dysregulation of microRNA expression in obese white adipose tissue. J Clin Endocrinol Metab. 2014;99(8):2821-33.

16. Mudhasani R, Puri V, Hoover K, Czech MP, Imbalzano AN, Jones SN. Dicer is required for the formation of white but not brown adipose tissue. J Cell Physiol. 2011;226(5):1399-406.

17. Ortega FJ, Mercader JM, Catalan V, Moreno-Navarrete JM, Pueyo N, Sabater $M$, et al. Targeting the circulating microRNA signature of obesity. Clin Chem. 2013;59(5):781-92.

18. Dankel SN, Fadnes DJ, Stavrum AK, Stansberg C, Holdhus R, Hoang T, et al. Switch from stress response to homeobox transcription factors in adipose tissue after profound fat loss. PLoS One. 2010;5(6):e11033.

19. Ogawa $R$, Tanaka C, Sato M, Nagasaki H, Sugimura K, Okumura K, et al. Adipocyte-derived microvesicles contain RNA that is transported into macrophages and might be secreted into blood circulation. Biochem Biophys Res Commun. 2010;398(4):723-9.

20. Mittelbrunn M, Gutierrez-Vazquez C, Villarroya-Beltri C, Gonzalez S, Sanchez-Cabo F, Gonzalez MA, et al. Unidirectional transfer of microRNA-loaded exosomes from T cells to antigen-presenting cells. Nat Commun. 2011;2:282.

21. Valadi H, Ekstrom K, Bossios A, Sjostrand M, Lee JJ, Lotvall JO. Exosomemediated transfer of mRNAs and microRNAs is a novel mechanism of genetic exchange between cells. Nat Cell Biol. 2007;9(6):654-9.

22. Thery C, Ostrowski M, Segura E. Membrane vesicles as conveyors of immune responses. NatRevImmunol. 2009;9(8):581-93.

23. Deng ZB, Poliakov A, Hardy RW, Clements R, Liu C, Liu Y, et al. Adipose tissue exosome-like vesicles mediate activation of macrophage-induced insulin resistance. Diabetes. 2009;58(11):2498-505.

24. Chartoumpekis DV, Zaravinos A, Ziros PG, Iskrenova RP, Psyrogiannis Al, Kyriazopoulou VE, et al. Differential expression of microRNAs in adipose tissue after long-term high-fat diet-induced obesity in mice. PLoS One. 2012;7(4):e34872.

25. Fernandez-Messina L, Gutierrez-Vazquez C, Rivas-Garcia E, Sanchez-Madrid F, de la Fuente $\mathrm{H}$. Immunomodulatory role of microRNAs transferred by extracellular vesicles. Biol Cell. 2015;107(3):61-77.
26. Nesca V, Guay C, Jacovetti C, Menoud V, Peyot ML, Laybutt DR, et al. Identification of particular groups of microRNAs that positively or negatively impact on beta cell function in obese models of type 2 diabetes. Diabetologia. 2013;56(10):2203-12.

27. LV YC, Tang YY, Peng J, Zhao GJ, Yang J, Yao F, et al. MicroRNA-19b promotes macrophage cholesterol accumulation and aortic atherosclerosis by targeting ATP-binding cassette transporter A1. Atherosclerosis. 2014;236(1):215-26.

28. Hulsmans M, De Keyzer D, Holvoet P. MicroRNAs regulating oxidative stress and inflammation in relation to obesity and atherosclerosis. Faseb J. 2011;25(8):2515-27.

29. O'Connell RM, Taganov KD, Boldin MP, Cheng G, Baltimore D. MicroRNA-155 is induced during the macrophage inflammatory response. Proc Natl Acad Sci U S A. 2007;104(5):1604-9.

30. Xie H, Lim B, Lodish HF. MicroRNAs induced during adipogenesis that accelerate fat cell development are downregulated in obesity. Diabetes. 2009;58(5):1050-7.

31. Papadopoulos GL, Alexiou P, Maragkakis M, Reczko M, Hatzigeorgiou AG. DIANA-mirPath: Integrating human and mouse microRNAs in pathways. Bioinformatics. 2009;25(15):1991-3.

32. McCurdy CE, Schenk S, Holliday MJ, Philp A, Houck JA, Patsouris D, et al. Attenuated Pik3r1 expression prevents insulin resistance and adipose tissue macrophage accumulation in diet-induced obese mice. Diabetes. 2012;61(10):2495-505.

33. Prada PO, Pauli JR, Ropelle ER, Zecchin HG, Carvalheira JB, Velloso LA, et al. Selective modulation of the CAP/Cbl pathway in the adipose tissue of high fat diet treated rats. FEBS Lett. 2006;580(20):4889-94.

34. Charriere G, Cousin B, Arnaud E, Andre M, Bacou F, Penicaud L, et al. Preadipocyte conversion to macrophage. Evidence of plasticity. J Biol Chem. 2003;278(11):9850-5.

35. Fain JN, Madan AK, Hiler ML, Cheema P, Bahouth SW. Comparison of the release of adipokines by adipose tissue, adipose tissue matrix, and adipocytes from visceral and subcutaneous abdominal adipose tissues of obese humans. Endocrinology. 2004;145(5):2273-82.

36. Cousin B, Munoz O, Andre M, Fontanilles AM, Dani C, Cousin JL, et al. A role for preadipocytes as macrophage-like cells. Faseb J. 1999;13(2):305-12.

37. Vandesompele J, De Preter K, Pattyn F, Poppe B, Van Roy N, De Paepe A, et al. Accurate normalization of real-time quantitative RT-PCR data by geometric averaging of multiple internal control genes. Genome Biol. 2002;3(7):RESEARCH0034.

38. Vergoulis T, Vlachos IS, Alexiou P, Georgakilas G, Maragkakis M, Reczko M, et al. TarBase 6.0: capturing the exponential growth of miRNA targets with experimental support. Nucleic Acids Res. 2012;40(Database issue):D222-9.

\section{Submit your next manuscript to BioMed Central and take full advantage of:}

- Convenient online submission

- Thorough peer review

- No space constraints or color figure charges

- Immediate publication on acceptance

- Inclusion in PubMed, CAS, Scopus and Google Scholar

- Research which is freely available for redistribution 